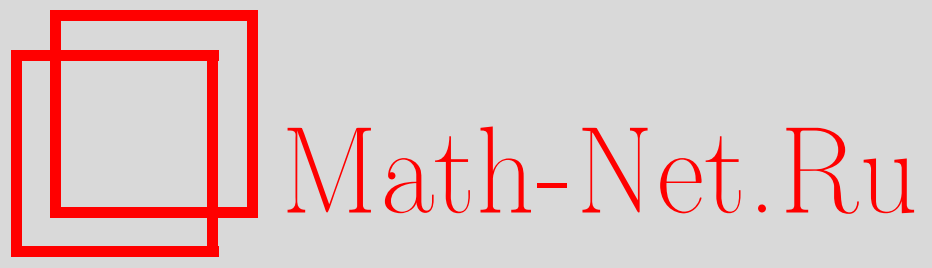

М. Н. Малышкин, Субэкспоненциальные оценки скорости сходимости к инвариантной мере для стохастических дифференциальных уравнений, Теория вероятн. и ее примен., 2000, том 45, выпуск 3, 489-504

DOI: https://doi.org/10.4213/tvp481

Использование Общероссийского математического портала Math-Net.Ru подразумевает, что вы прочитали и согласны с пользовательским соглашением http://www . mathnet.ru/rus/agreement

Параметры загрузки:

IP : 54.174 .149 .18

26 апреля 2023 г., $16: 25: 42$

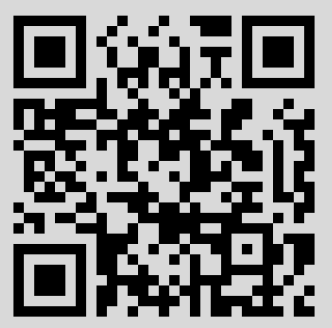


(c) 2000 r.

\author{
МАЛЫШКИН М. Н.*
}

\title{
СУБЭКСПОНЕНЦИАЛЬНЫЕ ОЦЕНКИ СКОРОСТИ СХОДИМОСТИ К ИНВАРИАНТНОЙ МЕРЕ ДЛЯ СТОХАСТИЧЕСКИХ ДИФФЕРЕНЦИАЛЬНЫХ УРАВНЕНИЙ
}

\begin{abstract}
В работе доказывается существование и единственность инвариантной меры для стохастического дифференциального уравнения, а также найдены условия на коэффициент сноса, обеспечивающие субэкспоненциальную скорость сходимости к инвариантной мере и субэкспоненциальную скорость сходимости коэффициентов перемешивания Колмогорова.

Ключевые слова и фразы: стохастические дифференциальные уравнения, инвариантная мера, коэффициенты перемешивания, субэкспоненциальная скорость сходимости.
\end{abstract}

1. Введение. Рассмотрим решение стохастического дифференциального уравнения

$$
d X_{t}=b\left(X_{t}\right) d t+\sigma\left(X_{t}\right) d w_{t}, \quad X_{0}=x \in \mathbf{R}^{d}
$$

где $w_{t}, t \geqslant 0$, - винеровский процесс, $b$ - измеримая, локально ограниченная функция, $\sigma$ - ограниченная, непрерывная, невырожденная матрица размера $d \times d$. Обозначим

$$
\begin{gathered}
a(x)=\sigma \sigma^{*}(x), \quad \Lambda=\sup _{x} \frac{\operatorname{Tr} a(x)}{d}, \\
\lambda_{+}=\sup _{x \neq 0}\left(a(x) \frac{x}{|x|}, \frac{x}{|x|}\right), \quad \lambda_{-}=\inf _{x \neq 0}\left(a(x) \frac{x}{|x|}, \frac{x}{|x|}\right) .
\end{gathered}
$$

Пусть выполнено условие:

$$
\left(b(x), \frac{x}{|x|}\right) \leqslant-\frac{r}{|x|^{p}}, \quad|x| \geqslant M_{0}>0,0<p<1, r>0 .
$$

${ }^{*}$ Московский государственный университет им. М.В. Ломоносова, механикоматематический фахультет, Воробьевы горы, 119899 Москва, Россия; е-mail: mnmal@mail.ru

1) Работа выполнена при финансовой поддержке Российского фонда фундаментальных исследований, грант 98-01-00431, и INTAS, грант 94-0378. 
Хорошо известно, что при выполнении условия $(b(x), x /|x|) \leqslant-r$ у процесса (1) существует инвариантная мера и справедлива оценка вида

$$
\mathbf{E}_{x} e^{c \tau}<\infty, \quad \text { где } \tau=\inf \left(t \geqslant 0:\left|X_{t}\right| \leqslant M\right) .
$$

Кроме того, имеет место экспоненциальная скорость сходимости к инвариантной мере (см. [3]). В работе [1] доказано, что для $r>(d / 2) \Lambda+$ $\lambda_{+}+\frac{1}{2}\left(\lambda_{+}-\lambda_{-}\right)$при выполнении условия

$$
\left(b(x), \frac{x}{|x|}\right) \leqslant-\frac{r}{|x|}, \quad|x| \geqslant M_{0},
$$

существуют инвариантная мера и оценки:

$$
\mathbf{E}_{x} \tau^{k} \leqslant C\left(1+|x|^{m}\right), \quad \operatorname{var}\left(\mu^{x}(t)-\mu^{\mathrm{inv}}\right) \leqslant C(x)(1+t)^{-k}, \quad k, m>0 .
$$

То есть имеется полиномиальная скорость сходимости к инвариантной мере. Так же оценивается скорость сходимости коэффициента перемешивания Колмогорова:

$$
\beta^{x}(t) \leqslant C(x)(1+t)^{-k},
$$

где $\beta^{x}(t)=\sup _{t \geqslant 0} \mathbf{E} \operatorname{var}_{B \in \mathscr{F}_{\geqslant t+s}^{X}}\left(\mathbf{P}\left(B \mid \mathscr{F}_{s}^{X}\right)-\mathbf{P}(B)\right), \mathscr{F}_{\boldsymbol{s}}^{X}=\sigma\left(X_{s^{\prime}}, s^{\prime} \leqslant s\right)$, $\mathscr{F}_{\geqslant t+s}^{X}=\sigma\left(X_{s^{\prime}}, s^{\prime} \geqslant t+s\right)$.

Похожие вопросы (с экспоненциальной и полиномиальной скоростью сходимости) изучались для дискретного времени (см. [9], [11]-[13], [15]).

В работе [10] получены результаты с субэкспоненциальной скоростью сходимости к инвариантной мере для стохастического дифференциального уравнения. Используется полугрупповой подход к решению данной проблемы. Отличие результатов данной работы в том, что мы налагаем более слабые ограничения на коэффициенты сноса и диффузии, а также используем другие методы решения. А именно, в данной статье мы применяем технику, основанную на возвратности процесса (и, более того, на конечности субэкспоненциальных моментов времени возвращения процесса). Также применяется метод склейки. Эта техника широко используется в [1], [2].

Кроме того, будет доказано существование и единственность инвариантой меры у процесса (1) при более слабом, чем в [1], условии, а именно, при условии (3) с $r>(d / 2) \Lambda$.

В данной работе мы используем результаты Крылова (см. [6], [7]) о существовании слабого решения уравнения (1) и его строгой марковости.

2. Сушествование и единственность инвариантной меры.

Лемма 1. При выполнении условия (3) $c r>(d / 2) \Lambda$, справедлива оченка: $\forall m \in\left(2,2+(2 r-d \Lambda) \lambda_{+}^{-1}\right)$

$$
\mathbf{E}_{x} \tau \leqslant C(m)\left(1+|x|^{m}\right) .
$$


Д ок аз а те льст во. Применим формулу Ито к процессу $(1+t)^{k}\left|X_{t}\right|^{m}$, при $k \in(-1,0]$ и $t<\tau$ получаем:

$$
\begin{aligned}
& d(1+t)^{k}\left|X_{t}\right|^{m}=(1+t)^{k}\left|X_{t}\right|^{m-2} \\
& \times\left[k(1+t)^{-1}\left|X_{t}\right|^{2}+m\left(X_{t}, b\left(X_{t}\right)\right)\right. \\
& \left.\quad+\frac{m(m-2)}{2}\left(a\left(X_{t}\right) \frac{X_{t}}{\left|X_{t}\right|}, \frac{X_{t}}{\left|X_{t}\right|}\right)+\frac{m}{2} \operatorname{Tr} a\left(X_{t}\right)\right] d t \\
& \quad+m(1+t)^{k}\left|X_{t}\right|^{m-2} \sum_{i, j=1}^{d} X_{t}^{i} \sigma_{i}^{j}\left(X_{t}\right) d w_{t}^{j} \\
& \leqslant(1+t)^{k}\left|X_{t}\right|^{m-2}\left[-r m+\frac{m(m-2)}{2} \lambda_{+}+\frac{m d}{2} \Lambda\right] d t \\
& +m(1+t)^{k}\left|X_{t}\right|^{m-2} \sum_{i, j=1}^{d} X_{t}^{i} \sigma_{i}^{j}\left(X_{t}\right) d w_{t}^{j} .
\end{aligned}
$$

Поэтому

$$
\begin{aligned}
& \mathbf{E}\left[(1+t)^{k}\left|X_{t}\right|^{m} 1(t<\tau)\right] \\
& \quad \leqslant-m \mathbf{E} \int_{0}^{t}(1+s)^{k}\left|X_{s}\right|^{m-2}\left(r-\frac{m-2}{2} \lambda_{+}-\frac{d}{2} \Lambda\right) 1(s<\tau) d s \equiv J .
\end{aligned}
$$

Так как $s<\tau, m \in\left(2,2+(2 r-d \Lambda) \lambda_{+}^{-1}\right)$, получаем $\left|X_{s}\right|^{m-2}>M_{0}^{m-2}$, $r-(m-2) \lambda_{+} / 2-d \Lambda / 2>0$, отсюда

$$
\begin{aligned}
J & \leqslant-m\left(r-\frac{m-2}{2} \lambda_{+}-\frac{d}{2} \Lambda\right) M_{0}^{m-2} \mathbf{E} \int_{0}^{t}(1+s)^{k} 1(s<\tau) d s \\
& =-\widehat{C}\left(\mathbf{E}(1+t \wedge \tau)^{k+1}-1\right), \quad \text { где } t \wedge \tau=\min (t, \tau) .
\end{aligned}
$$

Тогда $\hat{C} \mathbf{E}(1+t \wedge \tau)^{k+1} \leqslant \widehat{C}+|x|^{m}$. При $k=0$ для любого $t \geqslant 0$ получаем

$$
\mathbf{E}(1+t \wedge \tau) \leqslant C(m)\left(1+|x|^{m}\right) .
$$

Отсюда с помощью леммы Фату получаем (4).

Теорема 1. При выполнении условия (3) $c r>(d / 2) \Lambda$ существует и единственна инвариантная мера $\mu^{X, \text { inv }}$ для уравнения (1).

Д ок аз а тельст в о. Введем следующие области: $U=\{x$ : $|x|<M\}$, где $M>M_{0}, \Gamma=\partial U ; U_{1}=\{x:|x|<M+1\}, \Gamma_{1}=\partial U_{1}$.

Будем рассматривать последовательность моментов остановки: $\tau_{0}=0$,

$$
\tau_{1}^{\prime}=\inf \left\{t \geqslant \tau_{0} \mid X_{t} \in \Gamma\right\}, \quad \tau_{1}=\inf \left\{t \geqslant \tau_{1}^{\prime} \mid X_{t} \in \Gamma_{1}\right\}, \quad \ldots
$$


Из (4) следует, что $\sup _{x \in K} \mathbf{E}_{x} \tau_{1}^{\prime}<\infty$ для любого компакта $K \subset \bar{U}$, а по следствию 2 теоремы 3.7 .1 из [5] $\sup _{|x|=M} \mathbf{E}_{x} \tau_{1}<\infty$. Таким образом, все условия теоремы 4.4.1 из [5] выполнены. Кроме того, в [5] от коэффициентов уравнения требуется липшицевость. Как можно заметить, это не является препятствием: липшицевость требуется только в доказательстве существования положительной плотности у распределения $\mathbf{P}\left\{X\left(\tau_{U_{1}}\right) \in \gamma\right\}, \gamma \in \Gamma_{1}=\partial U_{1}$, но для доказательства существования плотности можно применить неравенство Харнака для параболического уравнения с измеримыми коэффициентами (см. [8]).

Применяя следствие 4.5.2 из [5], получаем единственность инвариантной меры.

3 а м е ч а н и е 1 . Как легко увидеть, условие (3) с $r>(d / 2) \Lambda$ слабее (2), поэтому утверждения теоремы 1 остаюося верными при условии (2).

\section{3. Субэкспоненциальные опенки.}

Теорема 2. При выполнении условия (2) $\forall \alpha \in(0,(1-p) / 2)$, $\beta \in[2 \alpha, 1-p), \exists \delta>0$ :

$$
\operatorname{var}\left(\mu^{x}(t)-\mu^{\mathrm{inv}}\right) \leqslant C\left(1+e^{\delta|x|^{\beta}}\right) e^{-\delta t^{\alpha}},
$$

где $\mu^{x}(t)$ - распределение $X_{t}, x=X_{0}, u \mu^{\mathrm{inv}}-$ инвариантная мера $\partial \Omega g X_{t}$.

При условии (2) с $r>(1-p) \lambda_{+} / 2$ утверждение теоремы выполняется $и$ dлs $\alpha=(1-p) / 2, \beta=1-p$.

Теорема 3. При выполнении условия (2) $\forall \alpha \in(0,(1-p) / 2)$, $\beta \in[2 \alpha, 1-p)$

$$
\mathbf{E}_{x} e^{\left|X_{t}\right|^{\beta}} \leqslant C\left(1+e^{|x|^{\beta}}\right) .
$$

При условии (2) с $r>(1-p) \lambda_{+} / 2$ утверждение теоремы выполняется $u \partial л g \alpha=(1-p) / 2, \beta=1-p$.

Теорема 4. При выполнении условий теоремы 2

$$
\beta^{x}(t) \leqslant C\left(1+e^{\delta|x|^{\beta}}\right) e^{-\delta t^{\alpha}} .
$$

4. Вспомогательные результаты.

Лемма 2. При выполнении условия (2) $\forall 0<\beta<1-p, k>0$

$$
\sup _{t \geqslant 0} \mathbf{E}_{x} e^{k\left|X_{t}\right|^{\beta}} 1(t<\tau) \leqslant e^{k|x|^{\beta}} .
$$

При условии (2) $с r>k(1-p) \lambda_{+} / 2$ утверждение теоремы выполняется $u \partial_{\Omega} \beta=1-p$. 
Д ок а з а т е л с т во. Применим формулу Ито к процессу $\exp \left(k\left|X_{t}\right|^{\beta}\right)$, при $t<\tau$ получаем:

$$
\begin{aligned}
d e^{k\left|X_{t}\right|^{\beta}=} & \frac{k \beta e^{k\left|X_{t}\right|^{\beta}}}{\left|X_{t}\right|^{2-\beta}}\left(\left(X_{t}, b\left(X_{t}\right)\right)+\frac{1}{2} \operatorname{Tr} a\left(X_{t}\right)\right. \\
& \left.\quad+\frac{1}{2}\left(a\left(X_{t}\right) \frac{X_{t}}{\left|X_{t}\right|}, \frac{X_{t}}{\left|X_{t}\right|}\right)\left(k \beta\left|X_{t}\right|^{\beta}+\beta-2\right)\right) d t \\
& +\sum_{i, j=1}^{d} k \beta e^{k\left|X_{t}\right|^{\beta}}\left|X_{t}\right|^{\beta-2} X_{t}^{i} \sigma_{i}^{j}\left(X_{t}\right) d w_{t}^{j} \\
\leqslant & \frac{k \beta e^{k\left|X_{t}\right|^{\beta}}}{\left|X_{t}\right|^{2-\beta}}\left(\left(X_{t}, b\left(X_{t}\right)\right)+\frac{d}{2} \Lambda+\frac{k \beta\left|X_{t}\right|^{\beta}}{2} \lambda_{+}-\frac{2-\beta}{2} \lambda_{-}\right) d t \\
& +\sum_{i, j=1}^{d} k \beta e^{k\left|X_{t}\right|^{\beta}}\left|X_{t}\right|^{\beta-2} X_{t}^{i} \sigma_{i}^{j}\left(X_{t}\right) d w_{t}^{j} .
\end{aligned}
$$

Отсюда

$$
\begin{aligned}
& \mathbf{E}_{x} e^{k\left|X_{t}\right|^{\beta}} 1(t<\tau)-e^{k|x|^{\beta}} \\
& \quad \leqslant \mathbf{E}_{x} \int_{0}^{t} \frac{k \beta e^{k\left|X_{s}\right|^{\beta}}}{\left|X_{s}\right|^{2-\beta}}\left(-r\left|X_{s}\right|^{1-p}+\frac{k \beta\left|X_{s}\right|^{\beta}}{2} \lambda_{+}+\frac{d}{2} \Lambda\right) 1(s<\tau) d s \leqslant 0
\end{aligned}
$$

(при достаточно большом $M \forall r>0, k>0$ т.к. при $\beta=1-p$ получаем, что $r>k(1-p) \lambda_{+} / 2$; это неравенство сохраняется и для $\left.\beta<1-p\right)$.

Отсюда сразу получаем (8).

Теорема 5. При выполнении условия (2) $\forall \alpha \in(0,(1-p) / 2), \beta \in$ $[2 \alpha, 1-p)$ существует $M_{1} \geqslant M_{0}$ maкое, что для любого $M \geqslant M_{1}$

$$
\mathbf{E}_{x} e^{\tau^{\alpha}} \leqslant C\left(1+e^{|x|^{\beta}}\right) .
$$

При условии (2) с $r>(1-p) \lambda_{+} / 2$ утверждение теоремы выполняется u $\partial \Omega я \alpha=(1-p) / 2, \beta=1-p$.

Д ок аз а те ль с т о. Применим формулу Ито к процессу $\left[e^{(1+t)^{\alpha}} /(1+t)^{1-\alpha}\right]\left|X_{t}\right|^{m}$, при $t<\tau, m \geqslant 2$ получаем:

$$
\begin{aligned}
& d \frac{e^{(1+t)^{\alpha}}}{(1+t)^{1-\alpha}}\left|X_{t}\right|^{m}=\frac{e^{(1+t)^{\alpha}}}{(1+t)^{2(1-\alpha)}}\left|X_{t}\right|^{m-2} \\
& \quad \times\left[\left(\alpha-\frac{1-\alpha}{(t+1)^{\alpha}}\right)\left|X_{t}\right|^{2}+m(t+1)^{1-\alpha}\right. \\
& \left.\quad \times\left(\left(X_{t}, b\left(X_{t}\right)\right)+\frac{1}{2}(m-2)\left(a\left(X_{t}\right) \frac{X_{t}}{\left|X_{t}\right|}, \frac{X_{t}}{\left|X_{t}\right|}\right)+\frac{1}{2} \operatorname{Tr} a\left(X_{t}\right)\right)\right] d t \\
& +m \frac{e^{(1+t)^{\alpha}}}{(1+t)^{1-\alpha}}\left|X_{t}\right|^{m-2} \sum_{i, j=1}^{d} X_{t}^{i} \sigma_{i}^{j}\left(X_{t}\right) d w_{t}^{j} \leqslant \frac{e^{(1+t)^{\alpha}}}{(1+t)^{2(1-\alpha)}}\left|X_{t}\right|^{m-2}
\end{aligned}
$$




$$
\begin{aligned}
& \times\left[\alpha\left|X_{t}\right|^{2}+m(t+1)^{1-\alpha}\left(\left(X_{t}, b\left(X_{t}\right)\right)+\frac{m-2}{2} \lambda_{+}+\frac{d}{2} \Lambda\right)\right] d t \\
& +m \frac{e^{(1+t)^{\alpha}}}{(1+t)^{1-\alpha}}\left|X_{t}\right|^{m-2} \sum_{i, j=1}^{d} X_{t}^{i} \sigma_{i}^{j}\left(X_{t}\right) d w_{t}^{j} .
\end{aligned}
$$

Тогда

$$
\begin{aligned}
& \mathbf{E}_{x} \frac{e^{(1+t)^{\alpha}}}{(1+t)^{1-\alpha}}\left|X_{t}\right|^{m} 1(t<\tau)-e|x|^{m} \\
& \leqslant \mathbf{E}_{x} \int_{0}^{t} \frac{e^{(1+s)^{\alpha}}}{(1+s)^{2(1-\alpha)}\left|X_{s}\right|^{m+\beta-2}\left[\alpha\left|X_{s}\right|^{2-\beta}-m(s+1)^{1-\alpha}\right.} \\
& \left.\quad \times\left(r\left|X_{s}\right|^{1-p-\beta}-\frac{1}{2\left|X_{s}\right|^{\beta}}\left((m-2) \lambda_{+}+d \Lambda\right)\right)\right] 1(s<\tau) d s \\
& \leqslant \mathbf{E}_{x} \int_{0}^{t} \frac{e^{(1+s)^{\alpha}}}{(1+s)^{2(1-\alpha)}}\left|X_{s}\right|^{m+\beta-2} \\
& \quad \times\left[\alpha\left|X_{s}\right|^{2-\beta}-m \varepsilon_{0}(s+1)^{1-\alpha}\right] 1(s<\tau) d s \equiv H
\end{aligned}
$$

При достаточно большом $M$

$$
\varepsilon_{0}=r M^{1-p-\beta}-\frac{(m-2) \lambda_{+}+\Lambda d}{2 M^{\beta}}>0 .
$$

\section{Отсюда}

$$
\begin{aligned}
H= & \mathbf{E}_{x} \int_{0}^{t} \frac{e^{(1+s)^{\alpha}}}{(1+s)^{2(1-\alpha)}}\left|X_{s}\right|^{m+\beta-2}\left[\alpha\left|X_{s}\right|^{2-\beta}-m \varepsilon_{0}(s+1)^{1-\alpha}\right] \\
& \times\left(1\left(\left|X_{s}\right|^{2-\beta} \leqslant \varepsilon_{1}(1+s)^{1-\alpha}\right)\right. \\
& \left.\quad+1\left(\left|X_{s}\right|^{2-\beta}>\varepsilon_{1}(1+s)^{1-\alpha}\right)\right) 1(s<\tau) d s \equiv H_{1}+H_{2}
\end{aligned}
$$

где $\varepsilon_{1}=m \varepsilon_{0} /(2 \alpha)$;

$$
\begin{aligned}
H_{1} \leqslant & -\varepsilon_{1} \alpha \mathbf{E}_{x} \int_{0}^{t} \frac{e^{(1+s)^{\alpha}}\left|X_{s}\right|^{m+\beta-2}}{(1+s)^{1-\alpha}} 1\left(\left|X_{s}\right|^{2-\beta} \leqslant \varepsilon_{1}(1+s)^{1-\alpha}\right) 1(s<\tau) d s \\
\leqslant & -\varepsilon_{1} \alpha M^{m+\beta-2} \mathbf{E}_{x} \int_{0}^{t} \frac{e^{(1+s)^{\alpha}}}{(1+s)^{1-\alpha}} 1(s<\tau) d s \\
& +\varepsilon_{1} \alpha \mathbf{E}_{x} \int_{0}^{t} \frac{e^{(1+s)^{\alpha}}\left|X_{s}\right|^{m+\beta-2}}{(1+s)^{1-\alpha}} 1\left(\left|X_{s}\right|^{2-\beta}>\varepsilon_{1}(1+s)^{1-\alpha}\right) 1(s<\tau) d s \\
= & -\varepsilon_{1} M^{m+\beta-2} \mathbf{E}_{x} e^{\left(\tau_{t}+1\right)^{\alpha}}+H_{3}, \quad \text { где } \tau_{t}=\tau \wedge t ;
\end{aligned}
$$




$$
\begin{aligned}
H_{2} \leqslant & \alpha \mathbf{E}_{x} \int_{0}^{t} \frac{e^{(1+s)^{\alpha}}\left|X_{s}\right|^{m}}{(1+s)^{2(1-\alpha)}} 1\left(\left|X_{s}\right|^{2-\beta}>\varepsilon_{1}(1+s)^{1-\alpha}\right) 1(s<\tau) d s \\
\leqslant & \int_{0}^{\infty} \frac{e^{(1+s)^{\alpha}}}{(1+s)^{2(1-\alpha)}}\left(\mathbf{E}_{x}\left|X_{s}\right|^{a m} 1(s<\tau)\right)^{1 / a} \\
& \times\left(\mathbf{P}_{x}\left\{\left|X_{s}\right|^{2-\beta}>\varepsilon_{1}(1+s)^{1-\alpha} ; s<\tau\right\}\right)^{1 / c} d s \\
\leqslant & \int_{0}^{\infty} \frac{e^{(1+s)^{\alpha}}}{(1+s)^{2(1-\alpha)}}\left(\mathbf{E}_{x} e^{\left|X_{s}\right|^{\beta}} 1(s<\tau)\right)^{1 / a} \\
& \times\left(\frac{\mathbf{E}_{x} e^{\left|X_{s}\right|^{\beta}} 1(s<\tau)}{\exp \left\{\varepsilon_{1}(1+s)^{(1-\alpha) \beta /(2-\beta)}\right\}}\right)^{1 / c} d s .
\end{aligned}
$$

При $c$, достаточно близком к 1 , получаем условие на $\beta:(1-\alpha) \beta /(2-\beta) \geqslant$ $\alpha$, отсюда $\beta \geqslant 2 \alpha$. Но по лемме $2: \beta \leqslant 1-p$, отсюда получаем условие $\alpha \in(0,(1-p) / 2]$, причем в случае $\alpha=(1-p) / 2$ величина $r$ из условия (2) должна быть строго больше $(1-p) \lambda_{+} / 2$. Тогда, применяя (8), получаем:

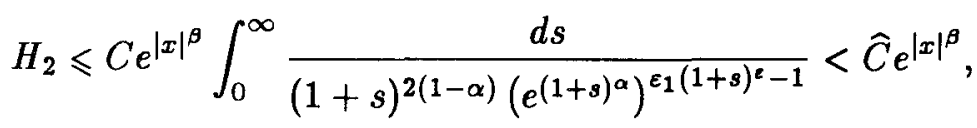

где $\varepsilon=(1-\alpha) \beta /(2-\beta)-\alpha \geqslant 0$. Аналогично показывается, что $H_{3} \leqslant$ $C e^{|x|^{\beta}}$. Отсюда

$$
\mathbf{E}_{x} e^{\tau_{t}^{\alpha}} \leqslant C(m)\left(1+e^{|x|^{\beta}}\right) .
$$

Чтобы получить (9), остается применить лемму Фату.

Лемма 3. При выполнении (2) $\forall 0<\beta<1-p, k>0$

$$
\int e^{k|x|^{\beta}} \mu(d x)<\infty
$$

где $\mu$ - инвариантная мера. При условии (2) с $r>k(1-p) \lambda_{+} / 2$ утверждение теоремы выполняется и для $\beta=1-p$.

Д ок а з а т е л с т в о. В [5] при доказательстве существования инвариантной меры получено ее представление:

$$
\int f(y) \mu(d y)=\frac{1}{\nu\left(\mathbf{R}^{d}\right)} \int_{\Gamma_{1}} \tilde{\mu}(d x) \mathbf{E}_{x} \int_{0}^{\tau_{1}} f(X(t)) d t
$$

где $\nu(A)=\int_{\Gamma_{1}} \tilde{\mu}(d x) \mathbf{E}_{x} \tau^{A}, \tilde{\mu}-$ стационарное распределение цепи Маркова, $\tau^{A}$ - время, проведенное $X(t)$ в множестве $A$ за первый цикл. Очевидно, $\tau^{A}<\tau_{1}$. Для доказательства (10) оценим:

$$
\begin{aligned}
\mathbf{E}_{x} \int_{0}^{\tau_{1}} e^{k\left|X_{s}\right|^{\beta}} d s= & \mathbf{E}_{x} \int_{0}^{\tau_{1}^{\prime}} e^{k\left|X_{s}\right|^{\beta}} d s+\mathbf{E}_{x} \int_{\tau_{1}^{\prime}}^{\tau_{1}} e^{k\left|X_{s}\right|^{\beta}} d s \\
\leqslant & \int_{0}^{\infty}\left(\mathbf{E}_{x} e^{a k\left|X_{s}\right|^{\beta}} 1\left(s<\tau_{1}^{\prime}\right)\right)^{1 / a}\left(\mathbf{P}_{x}\left\{\tau_{1}^{\prime}>s\right\}\right)^{1 / c} d s \\
& +e^{k(M+1)^{\beta}} \mathbf{E}_{x}\left(\tau_{1}-\tau_{1}^{\prime}\right) \equiv J
\end{aligned}
$$


При $\beta<1-p$ можно брать любые $a$ и $c$. При $\beta=1-p$ возьмем $a$ достаточно близким к единице. Тогда получаем условие на $r: r>k(1-$ p) $\lambda_{+} / 2$. Следовательно,

$$
\begin{aligned}
J & \leqslant C e^{k|x|^{\beta}}\left(1+e^{|x|^{\beta}}\right)^{1 / c} \int_{0}^{\infty} \frac{d s}{e^{s^{\alpha} / c}}+e^{k(M+1)^{\beta}} \mathbf{E}_{x}\left(\tau_{1}-\tau_{1}^{\prime}\right) \\
& \leqslant \widehat{C} e^{k|x|^{\beta}}\left(1+e^{|x|^{\beta}}\right)^{1 / c}+e^{k(M+1)^{\beta}} \mathbf{E}_{x}\left(\tau_{1}-\tau_{1}^{\prime}\right)
\end{aligned}
$$

Отсюда получаем:

$$
\begin{aligned}
\int e^{k|y|^{\beta}} \mu(d y) & \leqslant \frac{\widetilde{\mu}\left(\Gamma_{1}\right)}{\nu\left(\mathbf{R}^{d}\right)}\left(\sup _{|x|=M+1} \widehat{C} e^{k|x|^{\beta}}\left(1+e^{|x|^{\beta}}\right)^{1 / c}+\widetilde{C} \sup _{|x|=M} \mathbf{E}_{x} \tau_{1}\right) \\
& \leqslant C<\infty
\end{aligned}
$$

Лемма 3 доказана.

Рассмотрим пару $\left(X_{t}, Y_{t}\right)$ двух независимых решений уравнения (1) с начальными условиями $x$ и $y$ и двумя копиями винеровского процесса $w$ и $\widetilde{w}$ соответственно. Обозначим

$$
\gamma=\inf \left(t \geqslant 0:\left|X_{t}\right| \vee\left|Y_{t}\right| \leqslant M\right), \quad \text { где } a \vee b=\max (a, b) .
$$

Лемма 4. При выполнении условия (2) $\forall \alpha \in(0,(1-p) / 2), \beta \in$ $[2 \alpha, 1-p)$ существует $M_{1} \geqslant M_{0}$ такое, что для любого $M \geqslant M_{1}$

$$
\mathbf{E}_{x, y} e^{\gamma^{\alpha}} \leqslant C\left(1+e^{|x|^{\beta}}+e^{|y|^{\beta}}\right) .
$$

При условии (2) с $r>(1-p) \lambda_{+} / 2$ утверждение теоремы выполняется $u$ для $\alpha=(1-p) / 2, \beta=1-p$.

Д ок аз а тельство. Применим формулу Ито к процессу $\left[\left(e^{(1+t)^{\alpha}}\right) /(1+t)^{1-\alpha}\right]\left(\left|X_{t}\right|^{m}+\left|Y_{t}\right|^{m}\right)$, при $t<\gamma, m \geqslant 2$ получаем:

$$
\begin{aligned}
& d \frac{e^{(1+t)^{\alpha}}}{(1+t)^{1-\alpha}}\left(\left|X_{t}\right|^{m}+\left|Y_{t}\right|^{m}\right)=\frac{e^{(1+t)^{\alpha}}}{(1+t)^{2(1-\alpha)}} \\
& \quad \times\left\{| X _ { t } | ^ { m - 2 } \left[\left(\alpha-\frac{1-\alpha}{(t+1)^{\alpha}}\right)\left|X_{t}\right|^{2}+m(t+1)^{1-\alpha}\right.\right. \\
& \left.\quad \times\left(\left(X_{t}, b\left(X_{t}\right)\right)+\frac{1}{2}(m-2)\left(a\left(X_{t}\right) \frac{X_{t}}{\left|X_{t}\right|}, \frac{X_{t}}{\left|X_{t}\right|}\right)+\frac{1}{2} \operatorname{Tr} a\left(X_{t}\right)\right)\right] \\
& \quad+\left|Y_{t}\right|^{m-2}\left[\left(\alpha-\frac{1-\alpha}{(t+1)^{\alpha}}\right)\left|Y_{t}\right|^{2}+m(t+1)^{1-\alpha}\right. \\
& \left.\left.\quad \times\left(\left(Y_{t}, b\left(Y_{t}\right)\right)+\frac{1}{2}(m-2)\left(a\left(Y_{t}\right) \frac{Y_{t}}{\left|Y_{t}\right|}, \frac{Y_{t}}{\left|Y_{t}\right|}\right)+\frac{1}{2} \operatorname{Tr} a\left(Y_{t}\right)\right)\right]\right\} d t
\end{aligned}
$$




$$
\begin{aligned}
& +m \frac{e^{(1+t)^{\alpha}}}{(1+t)^{1-\alpha}}\left|X_{t}\right|^{m-2} \sum_{i, j=1}^{d} X_{t}^{i} \sigma_{i}^{j}\left(X_{t}\right) d w_{t}^{j} \\
& +m \frac{e^{(1+t)^{\alpha}}}{(1+t)^{1-\alpha}}\left|Y_{t}\right|^{m-2} \sum_{i, j=1}^{d} Y_{t}^{i} \sigma_{i}^{j}\left(Y_{t}\right) d \tilde{w}_{t}^{j} \leqslant \frac{e^{(1+t)^{\alpha}}}{(1+t)^{2(1-\alpha)}} \\
& \times\left\{\left|X_{t}\right|^{m-2}\left[\alpha\left|X_{t}\right|^{2}+m(t+1)^{1-\alpha}\left(\left(X_{t}, b\left(X_{t}\right)\right)+\frac{m-2}{2} \lambda_{+}+\frac{d}{2} \Lambda\right)\right]\right. \\
& \left.+\left|Y_{t}\right|^{m-2}\left[\alpha\left|Y_{t}\right|^{2}+m(t+1)^{1-\alpha}\left(\left(Y_{t}, b\left(Y_{t}\right)\right)+\frac{m-2}{2} \lambda_{+}+\frac{d}{2} \Lambda\right)\right]\right\} d t \\
& +m \frac{e^{(1+t)^{\alpha}}}{(1+t)^{1-\alpha}}\left|X_{t}\right|^{m-2} \sum_{i, j=1}^{d} X_{t}^{i} \sigma_{i}^{j}\left(X_{t}\right) d w_{t}^{j} \\
& +m \frac{e^{(1+t)^{\alpha}}}{(1+t)^{1-\alpha}}\left|Y_{t}\right|^{m-2} \sum_{i, j=1}^{d} Y_{t}^{i} \sigma_{i}^{j}\left(Y_{t}\right) d \tilde{w}_{t}^{j} .
\end{aligned}
$$

Обозначим $\gamma_{t}=\gamma \wedge t$. Тогда

$$
\begin{aligned}
& \mathbf{E}_{x, y} \frac{e^{(1+t)^{\alpha}}}{(1+t)^{1-\alpha}}\left(\left|X_{t}\right|^{m}+\left|Y_{t}\right|^{m}\right) 1(t<\gamma)-e\left(|x|^{m}+|y|^{m}\right) \\
& \leqslant \mathbf{E}_{x, y} \int_{0}^{\gamma_{t}} \frac{e^{(1+s)^{\alpha}}}{(1+s)^{2(1-\alpha)}} \\
& \times\left\{\left|X_{s}\right|^{m-2}\left[\alpha\left|X_{s}\right|^{2}+m(s+1)^{1-\alpha}\left(\left(X_{s}, b\left(X_{s}\right)\right)+\frac{m-2}{2} \lambda_{+}+\frac{d}{2} \Lambda\right)\right]\right. \\
& \left.+\left|Y_{s}\right|^{m-2}\left[\alpha\left|Y_{s}\right|^{2}+m(s+1)^{1-\alpha}\left(\left(Y_{s}, b\left(Y_{s}\right)\right)+\frac{m-2}{2} \lambda_{+}+\frac{d}{2} \Lambda\right)\right]\right\} \\
& \times\left\{1 \left(\left|X_{s}\right|^{2-\beta} \leqslant \varepsilon_{1}(1+s)^{1-\alpha} ;\left|X_{s}\right|>M ;\right.\right. \\
& \left.\left|Y_{s}\right|^{2-\beta} \leqslant \varepsilon_{1}(1+s)^{1-\alpha} ;\left|Y_{s}\right|>M\right) \\
& +1\left(\left|X_{s}\right|^{2-\beta} \leqslant \varepsilon_{1}(1+s)^{1-\alpha} ;\left|X_{s}\right|>M\right. \text {; } \\
& \left.\left|Y_{s}\right|^{2-\beta}>\varepsilon_{1}(1+s)^{1-\alpha} ;\left|Y_{s}\right|>M\right) \\
& +1\left(\left|X_{s}\right|^{2-\beta}>\varepsilon_{1}(1+s)^{1-\alpha} ;\left|X_{s}\right|>M\right. \text {; } \\
& \left.\left|Y_{s}\right|^{2-\beta} \leqslant \varepsilon_{1}(1+s)^{1-\alpha} ;\left|Y_{s}\right|>M\right) \\
& +1\left(\left|X_{s}\right|^{2-\beta}>\varepsilon_{1}(1+s)^{1-\alpha} ;\left|X_{s}\right|>M\right. \text {; } \\
& \left.\left|Y_{s}\right|^{2-\beta}>\varepsilon_{1}(1+s)^{1-\alpha} ;\left|Y_{s}\right|>M\right) \\
& +1\left(\left|X_{s}\right|^{2-\beta} \leqslant \varepsilon_{1}(1+s)^{1-\alpha} ;\left|X_{s}\right|>M ; M_{0} \leqslant\left|Y_{s}\right| \leqslant M\right)
\end{aligned}
$$




$$
\begin{aligned}
& +1\left(\left|X_{s}\right|^{2-\beta}>\varepsilon_{1}(1+s)^{1-\alpha} ;\left|X_{s}\right|>M ; M_{0} \leqslant\left|Y_{s}\right| \leqslant M\right) \\
& +1\left(M_{0} \leqslant\left|X_{s}\right| \leqslant M ;\left|Y_{s}\right|^{2-\beta}>\varepsilon_{1}(1+s)^{1-\alpha} ;\left|Y_{s}\right|>M\right) \\
& +1\left(M_{0} \leqslant\left|X_{s}\right| \leqslant M ;\left|Y_{s}\right|^{2-\beta} \leqslant \varepsilon_{1}(1+s)^{1-\alpha} ;\left|Y_{s}\right|>M\right) \\
& +1\left(\left|X_{s}\right|^{2-\beta}>\varepsilon_{1}(1+s)^{1-\alpha} ;\left|X_{s}\right|>M ;\left|Y_{s}\right|<M_{0}\right) \\
& +1\left(\left|X_{s}\right|^{2-\beta} \leqslant \varepsilon_{1}(1+s)^{1-\alpha} ;\left|X_{s}\right|>M ;\left|Y_{s}\right|<M_{0}\right) \\
& +1\left(\left|X_{s}\right|<M_{0} ;\left|Y_{s}\right|^{2-\beta}>\varepsilon_{1}(1+s)^{1-\alpha} ;\left|Y_{s}\right|>M\right) \\
& \left.+1\left(\left|X_{s}\right|<M_{0} ;\left|Y_{s}\right|^{2-\beta} \leqslant \varepsilon_{1}(1+s)^{1-\alpha} ;\left|Y_{s}\right|>M\right)\right\} d s \equiv \sum_{l=1}^{12} J_{l} .
\end{aligned}
$$

Здесь $\varepsilon_{1}$ такое же, как в доказательстве теоремы 5. Обозначим $I_{l}-$ индикатор, соответствующий интегралу $J_{l}$. Оценим интегралы:

$$
\begin{aligned}
& J_{1} \leqslant-\varepsilon_{1} \alpha M^{m+\beta-2} \mathbf{E}_{x, y} \int_{0}^{\gamma_{t}} \frac{e^{(1+s)^{\alpha}}}{(1+s)^{1-\alpha}} I_{1} d s ; \\
& J_{2} \leqslant-\varepsilon_{1} \alpha M^{m+\beta-2} \mathbf{E}_{x, y} \int_{0}^{\gamma_{t}} \frac{e^{(1+s)^{\alpha}}}{(1+s)^{1-\alpha}} I_{2} d s+J_{13} ; \\
& J_{3} \leqslant-\varepsilon_{1} \alpha M^{m+\beta-2} \mathbf{E}_{x, y} \int_{0}^{\gamma_{t}} \frac{e^{(1+s)^{\alpha}}}{(1+s)^{1-\alpha}} I_{3} d s+J_{14} ; \\
& J_{4} \leqslant J_{15}+J_{16} \\
& J_{5} \leqslant-\varepsilon_{1} \alpha M^{m+\beta-2} \mathbf{E}_{x, y} \int_{0}^{\gamma_{t}} \frac{e^{(1+s)^{\alpha}}}{(1+s)^{1-\alpha}} I_{5} d s \\
& +\mathbf{E}_{x, y} \int_{0}^{\gamma_{t}} \frac{e^{(1+s)^{\alpha}}}{(1+s)^{2(1-\alpha)}}\left|Y_{s}\right|^{m+\beta-2} \\
& \times\left[\alpha\left|Y_{s}\right|^{2-\beta}-m(s+1)^{1-\alpha}\right. \\
& \left.\times\left(r\left|Y_{s}\right|^{1-p-\beta}-\frac{(m-2) \lambda_{+}+\Lambda d}{2\left|Y_{s}\right|^{\beta}}\right)\right] I_{5} d s \\
& \leqslant-\varepsilon_{1} \alpha M^{m+\beta-2} \mathbf{E}_{x, y} \int_{0}^{\gamma_{t}} \frac{e^{(1+s)^{\alpha}}}{(1+s)^{1-\alpha}} I_{5} d s \\
& +\mathbf{E}_{x, y} \int_{0}^{\gamma_{t}} \frac{e^{(1+s)^{\alpha}}}{(1+s)^{2(1-\alpha)}}\left|Y_{s}\right|^{m+\beta-2}\left[\alpha\left|Y_{s}\right|^{2-\beta}-\tilde{\varepsilon}_{0} m(s+1)^{1-\alpha}\right] \\
& \times I_{5} 1\left(\left|Y_{s}\right|^{2-\beta}>\tilde{\varepsilon}_{1}(1+s)^{1-\alpha}\right) d s .
\end{aligned}
$$

Здесь

$$
\tilde{\varepsilon}_{0}=r M_{0}{ }^{1-p-\beta}-\frac{(m-2) \lambda_{+}+\Lambda d}{2 M_{0}{ }^{\beta}}>0 \quad \text { и } \quad \tilde{\varepsilon}_{1}=\frac{m \tilde{\varepsilon}_{0}}{2 \alpha}
$$


При достаточно большом $M_{0} \tilde{\varepsilon}_{0}>0$. Тогда

$$
\begin{aligned}
J_{5} \leqslant & -\varepsilon_{1} \alpha M^{m+\beta-2} \mathbf{E}_{x, y} \int_{0}^{\gamma_{t}} \frac{e^{(1+s)^{\alpha}}}{(1+s)^{1-\alpha}} I_{5} d s \\
& +M^{m} \int_{1}^{\left(M^{2-\beta} / \tilde{\varepsilon}_{1}\right)^{\alpha /(1-\alpha)}} e^{t} d t \\
\leqslant & -\varepsilon_{1} \alpha M^{m+\beta-2} \mathbf{E}_{x, y} \int_{0}^{\gamma_{t}} \frac{e^{(1+s)^{\alpha}}}{(1+s)^{1-\alpha}} I_{5} d s+M^{m} e^{\left(M^{2-\beta} / \tilde{\varepsilon}_{1}\right)^{\alpha /(1-\alpha)}}
\end{aligned}
$$

Аналогично оцениваем $J_{l}, l=6, \ldots, 12$ :

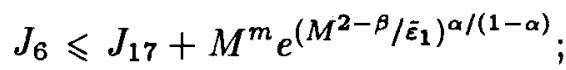

$$
\begin{aligned}
& J_{7} \leqslant M^{m} e^{\left(M^{2-\beta} / \tilde{\varepsilon}_{1}\right)^{\alpha /(1-\alpha)}}+J_{18} \\
& J_{8} \leqslant M^{m} e^{\left(M^{2-\beta} / \tilde{\varepsilon}_{1}\right)^{\alpha /(1-\alpha)}}-\varepsilon_{1} \alpha M^{m+\beta-2} \mathbf{E}_{x, y} \int_{0}^{\gamma_{t}} \frac{e^{(1+s)^{\alpha}-1}}{(1+s)^{1-\alpha}} I_{8} d s ; \\
& J_{9} \leqslant J_{19}+C M_{0}^{m} \mathbf{E}_{x, y} e^{\left(\gamma_{t}+1\right)^{\alpha}} \\
& J_{10} \leqslant-\varepsilon_{1} \alpha M^{m+\beta-2} \mathbf{E}_{x, y} \int_{0}^{\gamma_{t}} \frac{e^{(1+s)^{\alpha}}}{(1+s)^{1-\alpha}} I_{10} d s+C M_{0}^{m} \mathbf{E}_{x, y} e^{\left(\gamma_{t}+1\right)^{\alpha}} ; \\
& J_{11} \leqslant C M_{0}^{m} \mathbf{E}_{x, y} e^{\left(\gamma_{t}+1\right)^{\alpha}}+J_{20} \\
& J_{12} \leqslant C M_{0}^{m} \mathbf{E}_{x, y} e^{\left(\gamma_{t}+1\right)^{\alpha}}-\varepsilon_{1} \alpha M^{m+\beta-2} \mathbf{E}_{x, y} \int_{0}^{\gamma_{t}} \frac{e^{(1+s)^{\alpha}}}{(1+s)^{1-\alpha}} I_{12} d s . \\
& \sum_{l=1}^{12} J_{l} \leqslant C_{2}(M)+\sum_{l=13}^{20} J_{l}+C_{3} M_{0}^{m} \mathbf{E}_{x, y} e^{\left(\gamma_{t}+1\right)^{\alpha}} \\
& -C_{4} \alpha M^{m+\beta-2} \mathbf{E}_{x, y} \int_{0}^{\gamma_{t}} \frac{e^{(1+s)^{\alpha}}}{(1+s)^{1-\alpha}} \\
& \times\left(1-1\left(\left|X_{s}\right|^{2-\beta} \wedge\left|Y_{s}\right|^{2-\beta}>\varepsilon_{1}(1+s)^{1-\alpha}\right)\right) d s \\
& \equiv C_{2}(M)+\sum_{l=13}^{20} J_{l}+C_{3} M_{0}^{m} \mathbf{E}_{x, y} e^{\left(\gamma_{t}+1\right)^{\alpha}} \\
& -C_{4} M^{m+\beta-2} \mathbf{E}_{x, y} e^{\left(\gamma_{t}+1\right)^{\alpha}}+J_{21} .
\end{aligned}
$$

Интегралы $J_{l}, l=13, \ldots, 21$, оцениваются полностью аналогично интегралам $\mathrm{H}_{2}, \mathrm{H}_{3}$ из теоремы 5:

$$
\sum_{l=13}^{21} J_{l} \leqslant C\left(e^{|x|^{\beta}}+e^{|y|^{\beta}}\right) .
$$

При достаточно большом $M$ получаем $C_{4} M^{m+\beta-2}>C_{3} M_{0}^{m}$, тогда:

$$
\left(C_{4} M^{m+\beta-2}-C_{3} M_{0}^{m}\right) \mathbf{E}_{x, y} e^{\left(\gamma_{t}+1\right)^{\alpha}} \leqslant \widehat{C}\left(1+e^{|x|^{\beta}}+e^{|y|^{\beta}}\right) .
$$

Отсюда по лемме Фату получаем (11). Лемма 4 доказана. 
5. Доказательство субэкспоненциальных оценок.

Д ок а зательст во т е оремы 2. Доказательство этой теоремы аналогично доказательству теоремы 1 из [1]. Рассмотрим пару $\left(X_{t}, Y_{t}\right)$ двух независимых решений уравнения (1) с начальными значениями $X_{0}=x, Y_{0}$ имеет распределение $\mu^{\text {inv }}$. Введем последовательность моментов остановки:

$$
\gamma_{1}=\inf \left(t \geqslant s_{0}:\left|X_{t}\right| \vee\left|Y_{t}\right| \leqslant M\right) ;
$$

для $n \geqslant 1$

$$
\begin{aligned}
T_{n} & =\inf \left(t \geqslant \gamma_{n}:\left|X_{t}\right| \geqslant M+1 \text { или }\left|Y_{t}\right| \geqslant M+1\right), \\
T_{n}^{\prime} & =T_{n} \wedge\left(\gamma_{n}+1\right), \\
\gamma_{n+1} & =\inf \left(t \geqslant T_{n}^{\prime}:\left|X_{t}\right| \vee\left|Y_{t}\right| \leqslant M\right) .
\end{aligned}
$$

По лемме 4,

$$
\mathbf{E}\left(e^{\left(\gamma_{1}-s_{0}\right)^{\alpha}} \mid \widehat{\mathscr{F}}_{s_{0}}\right) \leqslant C \mathbf{E}\left(1+e^{\left|X_{s_{0}}\right|^{\beta}}+e^{\left|Y_{s_{0}}\right|^{\beta}} \mid \widehat{\mathscr{F}}_{s_{0}}\right),
$$

где $\widehat{\mathscr{F}}_{s_{0}} \equiv \mathscr{F}_{s_{0}}^{X, Y, \tilde{X}}$. При $s_{0}=0$, по лемме 3 :

$$
\mathbf{E}_{x} e^{\gamma_{1}^{\alpha}} \leqslant C \mathbf{E}_{x}\left(1+e^{|x|^{\beta}}+e^{\left|Y_{0}\right|^{\beta}}\right) \leqslant \hat{C}\left(1+e^{|x|^{\beta}}\right),
$$

аналогично,

$$
\begin{aligned}
\mathbf{E}\left(e^{\left(\gamma_{k}-T_{k-1}^{\prime}\right)^{\alpha}} \mid \widehat{\mathscr{F}}_{\gamma_{k-1}}\right) & \leqslant C \mathbf{E}\left(1+e^{\left|X_{T_{k-1}^{\prime}}\right|^{\beta}}+e^{\left|Y_{T_{k-1}^{\prime}}\right|^{\beta}} \mid \widehat{\mathscr{F}}_{\gamma_{k-1}}\right) \\
& \leqslant C\left(1+e^{(M+1)^{\beta}}\right)=\tilde{C} .
\end{aligned}
$$

Обозначим $n(t)=\sup \left(n \geqslant 0: \gamma_{n} \leqslant t\right)$. С помощью последнего неравенства и строго марковского свойства получаем

$$
\mathbf{P}\{n(t) \rightarrow \infty, t \rightarrow \infty\}=1 .
$$

Используя метод склейки (coupling method, см. [16], [4]) определим новый процесс $\left(\tilde{X}_{t}\right)$ и случайную величину $L=L_{s_{0}} \geqslant s_{0}$ на некотором расширении вероятностного пространства (при этом определения вероятности и математического ожидания не меняются). По определению $\left(\widetilde{X}_{t}\right)$ эквивалентен по распределению процессу $\left(X_{t}\right)$ и, более того,

$$
\mathbf{P}\left\{\tilde{X}_{t}=X_{t}, t \leqslant L_{s_{0}}\right\}=\mathbf{P}\left\{\tilde{X}_{t}=Y_{t}, t \geqslant L_{s_{0}}\right\}=1,
$$

где $L_{s_{0}}$ есть $\widehat{\mathscr{F}}_{s_{0}}$-измеримый момент остановки. Кроме того, существует $q \in(0,1)$ такое, что

$$
\sup _{s_{0} \geqslant 0} \mathbf{P}\left\{L_{s_{0}}>\gamma_{n} \mid \hat{\mathscr{F}}_{s_{0}}\right\} \leqslant q^{n} \quad \forall n .
$$


Для доказательства (5) заметим, что

$$
\operatorname{var}\left(\mu^{x}(t)-\mu^{\text {inv }}\right) \leqslant \sup _{A}\left|\mathbf{P}_{x}\left\{\tilde{X}_{t} \in A\right\}-\mathbf{P}\left\{Y_{t} \in A\right\}\right| \leqslant \mathbf{P}\left\{L_{0}>t\right\} .
$$

Тогда

$$
\begin{aligned}
\mathbf{P}_{x}\left\{L_{0}>t\right\} & =\sum_{n=0}^{\infty} \mathbf{E}_{x}\left(1\left(L_{0}>t\right) 1\left(\gamma_{n} \leqslant t<\gamma_{n+1}\right)\right) \\
& \leqslant \sum_{n=0}^{\infty}\left[\mathbf{P}_{x}\left\{L_{0}>\gamma_{n}\right\}\right]^{1 / a}\left[\mathbf{P}\left\{\gamma_{n+1}>t\right\}\right]^{1 / c}
\end{aligned}
$$

Как мы уже знаем, $\left[\mathbf{P}_{x}\left\{L_{0}>\gamma_{n}\right\}\right]^{1 / a} \leqslant\left(q^{1 / a}\right)^{n}$. По неравенству Чебышева при $0<\delta<1$ получаем

$$
\mathbf{P}_{x}\left\{\gamma_{n+1}>t\right\} \leqslant e^{-\delta t^{\alpha}} \mathbf{E}_{x} e^{\delta \gamma_{n+1}^{\alpha}}
$$

По неравенству Гёльдера:

$$
\mathbf{E}_{x} e^{\delta \gamma_{n+1}^{\alpha}} \leqslant\left(\mathbf{E}_{x} e^{(1 / \delta) \delta \gamma_{n+1}^{\alpha}}\right)^{\delta}\left(\mathbf{E}_{x} 1\right)^{1-\delta} \leqslant\left(\mathbf{E}_{x} e^{\gamma_{n+1}^{\alpha}}\right)^{\delta}
$$

Как мы уже знаем,

$$
\mathbf{E}_{x} e^{\gamma_{1}^{\alpha}} \leqslant C\left(1+e^{|x|^{\beta}}\right)
$$

Тогда, поскольку $\alpha<1$ :

$$
\begin{aligned}
\mathbf{E}_{x} e^{\gamma_{2}^{\alpha}} & =\mathbf{E}_{x} e^{\left(\gamma_{2}-T_{1}^{\prime}+T_{1}^{\prime}-\gamma_{1}+\gamma_{1}\right)^{\alpha}} \leqslant \mathbf{E}_{x} e^{\left(\gamma_{2}-T_{1}^{\prime}\right)^{\alpha}} e^{\left(T_{1}^{\prime}-\gamma_{1}\right)^{\alpha}} e^{\gamma_{1}^{\alpha}} \\
& \leqslant e \mathbf{E}_{x} e^{\left(\gamma_{2}-T_{1}^{\prime}\right)^{\alpha}} e^{\gamma_{1}^{\alpha}}=e \mathbf{E}_{x}\left(\mathbf{E}\left(e^{\left(\gamma_{2}-T_{1}^{\prime}\right)^{\alpha}} e^{\gamma_{1}^{\alpha}} \mid \widehat{\mathscr{F}}_{\gamma_{1}}\right)\right) \\
& =e \mathbf{E}_{x}\left(e^{\gamma_{1}^{\alpha}} \mathbf{E}\left(e^{\left(\gamma_{2}-T_{1}^{\prime}\right)^{\alpha}} \mid \widehat{\mathscr{F}}_{\gamma_{1}}\right)\right) \leqslant C\left(1+e^{(M+1)^{\beta}}\right)\left(1+e^{|x|^{\beta}}\right)
\end{aligned}
$$

По индукции,

$$
\begin{aligned}
\mathbf{E}_{x} e^{\gamma_{n+1}^{\alpha}} & =\mathbf{E}_{x} e^{\left(\gamma_{n+1}-T_{n}^{\prime}+T_{n}^{\prime}-\gamma_{n}+\gamma_{n}\right)^{\alpha}} \leqslant e \mathbf{E}_{x} e^{\left(\gamma_{n+1}-T_{n}^{\prime}\right)^{\alpha}} e^{\gamma_{n}^{\alpha}} \\
& =e \mathbf{E}_{x}\left(e^{\gamma_{n}^{\alpha}} \mathbf{E}\left(e^{\left(\gamma_{n+1}-T_{n}^{\prime}\right)^{\alpha}} \mid \widehat{\mathscr{F}}_{\gamma_{n}}\right)\right) \\
& \leqslant C^{n}\left(1+e^{(M+1)^{\beta}}\right)^{n}\left(1+e^{|x|^{\beta}}\right)
\end{aligned}
$$

Отсюда, при $c$, близком к единице, и достаточно малом $\delta$ (таком, что $\left.\widehat{C}^{\delta / c} q^{1 / a}=C^{\delta / c}\left(1+e^{(M+1)^{\beta}}\right)^{\delta / c} q^{1 / a}<1\right)$ получаем:

$\mathbf{P}_{x}\left\{L_{0}>t\right\}=e^{-\delta t^{\alpha} / c}\left(1+e^{|x|^{\beta}}\right)^{\delta / c} \sum_{n=0}^{\infty}\left(q^{1 / a}\right)^{n}\left(\widehat{C}^{\delta / c}\right)^{n} \leqslant C\left(1+e^{\delta|x|^{\beta}}\right) e^{-\delta t^{\alpha}}$

Таким образом, оценка (5), а вместе с ней и теорема 2 доказаны. 
Обозначим $\forall B \in \widehat{\mathscr{F}}_{t}$ $\mu^{\beta}(B)=\mathbf{E}\left(e^{\left|Y_{t}\right|^{\beta}} 1\left(Y_{t} \in B\right)\right), \quad \mu_{t}^{x, \beta}(B)=\mathbf{E}_{x}\left(e^{\left|X_{t}\right|^{\beta}} 1\left(X_{t} \in B\right)\right)$.

Лемма 5. При выполнении условий теоремы 2

$$
\operatorname{var}\left(\mu_{t}^{x, \beta}-\mu^{\beta}\right) \leqslant C\left(1+e^{\delta|x|^{\beta}}\right) e^{-\delta t^{\alpha}} .
$$

Доказ ате льство. Тогда

$$
\begin{aligned}
\left|\mu_{t}^{x, \beta}(B)-\mu^{\beta}(B)\right|= & \left|\mathbf{E}_{x}\left(e^{\left|X_{t}\right|^{\beta}} 1\left(X_{t} \in B\right)\right)-\mathbf{E}\left(e^{\left|Y_{t}\right|^{\beta}} 1\left(Y_{t} \in B\right)\right)\right| \\
= & \left|\mathbf{E}_{x}\left(e^{\left|\tilde{X}_{t}\right|^{\beta}} 1\left(\tilde{X}_{t} \in B\right)\right)-\mathbf{E}\left(e^{\left|Y_{t}\right|^{\beta}} 1\left(Y_{t} \in B\right)\right)\right| \\
\leqslant & \mathbf{E}_{x}\left(e^{\left|\tilde{X}_{t}\right|^{\beta}} 1\left(\tilde{X}_{t} \in B\right) 1\left(t<L_{0}\right)\right) \\
& +\mathbf{E}\left(e^{\left|Y_{t}\right|^{\beta}} 1\left(Y_{t} \in B\right) 1\left(t<L_{0}\right)\right) .
\end{aligned}
$$

По лемме 2 и теореме 2 ,

$$
\begin{aligned}
\mathbf{E}\left(e^{\left|Y_{t}\right|^{\beta}} 1\left(Y_{t} \in B\right) 1\left(t<L_{0}\right)\right) & \leqslant\left(\mathbf{E} e^{a\left|Y_{t}\right|^{\beta}}\right)^{1 / a}\left(\mathbf{P}\left\{L_{0}>t\right\}\right)^{1 / c} \\
& \leqslant C e^{-\delta t^{\alpha} / c}\left(1+e^{\delta|x|^{\beta} / c}\right)
\end{aligned}
$$

причем при $a$, близком к 1 , получаем условие на $r$ из леммы $2: r>$ $(1-p) \lambda_{+} / 2$. При этом

$$
\begin{aligned}
& \mathbf{E}_{x}\left(e^{\left|\tilde{X}_{t}\right|^{\beta}} 1\left(\tilde{X}_{t} \in B\right) 1\left(t<L_{0}\right)\right) \\
& \quad \leqslant \mathbf{E}_{x}\left(e^{\left|\tilde{X}_{t}\right|^{\beta}} \sum_{i=0}^{\infty} 1\left(\gamma_{i} \leqslant t<\gamma_{i+1}\right) 1\left(\gamma_{i}<L_{0}\right) 1\left(t<L_{0}\right)\right) .
\end{aligned}
$$

Кроме того,

$$
\begin{aligned}
& \mathbf{E}_{x}\left(e^{\left|\widetilde{X}_{t}\right|^{\beta}} 1\left(\gamma_{i} \leqslant t<\gamma_{i+1}\right) 1\left(\gamma_{i}<L_{0}\right) 1\left(t<L_{0}\right)\right) \\
& \leqslant\left(\mathbf{E}_{x} e^{a\left|\widetilde{X}_{t}\right|^{\beta}} 1\left(\gamma_{i} \leqslant t<\gamma_{i+1}\right)\right)^{1 / a}\left[\mathbf{P}_{x}\left\{\gamma_{i}<L_{0}\right\}\right]^{1 / b}\left[\mathbf{P}_{x}\left\{t<L_{0}\right\}\right]^{1 / c} \\
& \leqslant C e^{(M+1)^{\beta}} q^{i / b} e^{-\delta t^{\alpha} / c}\left(1+e^{\delta|x|^{\beta} / c}\right) \leqslant C e^{-\delta t^{\alpha} / c}\left(1+e^{\delta|x|^{\beta} / c}\right)(\widetilde{q})^{i},
\end{aligned}
$$

где $1 / a+1 / b+1 / c=1, a$ близко к $1, \tilde{q}<1$, поэтому

$$
\begin{aligned}
\mathbf{E}_{x}\left(e^{\left|\tilde{X}_{t}\right|^{\beta}} 1\left(\tilde{X}_{t} \in B\right) 1\left(t<L_{0}\right)\right) & \leqslant \sum_{i=0}^{\infty} C e^{-\delta t^{\alpha} / c}\left(1+e^{\delta|x|^{\beta} / c}\right)(\widetilde{q})^{i} \\
& \leqslant \widetilde{C} e^{-\delta t^{\alpha} / c}\left(1+e^{\delta|x|^{\beta} / c}\right) .
\end{aligned}
$$


Тогда сушествует $\delta>0$ такое, что

$$
\operatorname{var}\left(\mu_{t}^{x, \beta}-\mu^{\beta}\right) \leqslant C e^{-\delta t^{\alpha}}\left(1+e^{\delta|x|^{\beta}}\right) .
$$

Лемма 5 доказана.

Доказательст в о те оремы 3 . По теореме 2 и леммам 3 и 5 , получаем

$$
\begin{aligned}
\operatorname{var} \mu_{t}^{x, \beta} & \leqslant \operatorname{var} \mu^{\beta}+\operatorname{var}\left(\mu_{t}^{x, \beta}-\mu^{\beta}\right) \leqslant \widehat{C}+\widetilde{C} e^{-\delta t^{\alpha}}\left(1+e^{\delta|x|^{\beta}}\right) \\
& \leqslant C\left(1+e^{|x|^{\beta}}\right)
\end{aligned}
$$

Тогда $\forall t>0$ получаем (6):

$$
\mathbf{E}_{x} e^{\left|X_{t}\right|^{\beta}} \leqslant \sup _{B}\left|\mathbf{E}_{x} e^{\left|X_{t}\right|^{\beta}} 1\left(X_{t} \in B\right)\right|=\operatorname{var} \mu_{t}^{x, \beta} \leqslant C\left(1+e^{|x|^{\beta}}\right) .
$$

Д оказ ат тл вс т в о те о ре мы 4. Итак, $\forall B \in \widehat{\mathscr{F}}_{\geqslant t+s_{0}}$

$$
\begin{aligned}
\left|\mathbf{P}\left(B \mid \widehat{\mathscr{F}}_{s_{0}}\right)-\mathbf{P}(B)\right| & \leqslant\left|\mathbf{E}\left(1\left(L_{s_{0}}>t+s_{0}\right) \mid \widehat{\mathscr{F}}_{s_{0}}\right)\right| \\
& \leqslant \mathbf{P}\left\{L_{s_{0}}>t+s_{0} \mid \widehat{\mathscr{F}}_{s_{0}}\right\} .
\end{aligned}
$$

Torда

$$
\beta^{x}(t) \leqslant \mathbf{E}_{x} \mathbf{P}\left\{L_{s_{0}}>t+s_{0} \mid \widehat{\mathscr{F}}_{s_{0}}\right\}=\mathbf{P}\left\{L_{s_{0}}>t+s_{0}\right\}
$$

Кроме того,

$$
\begin{aligned}
\mathbf{P} & \left\{L_{s_{0}}>t+s_{0} \mid \widehat{\mathscr{F}}_{s_{0}}\right\} \\
& =\sum_{n=0}^{\infty} \mathbf{E}\left(1\left(L_{s_{0}}>t+s_{0}\right) 1\left(\gamma_{n} \leqslant t+s_{0}<\gamma_{n+1}\right) \mid \widehat{\mathscr{F}}_{s_{0}}\right) \\
& \leqslant \sum_{n=0}^{\infty}\left[\mathbf{P}\left\{L_{s_{0}}>\gamma_{n} \mid \widehat{\mathscr{F}}_{s_{0}}\right\}\right]^{1 / a}\left[\mathbf{P}\left\{\gamma_{n+1}>t+s_{0} \mid \widehat{\mathscr{F}}_{s_{0}}\right\}\right]^{1 / c} .
\end{aligned}
$$

Как мы уже знаем, $\left[\mathbf{P}\left\{L_{s_{0}}>\gamma_{n} \mid \widehat{\mathscr{F}}_{s_{0}}\right\}\right]^{1 / a} \leqslant\left(q^{1 / a}\right)^{n}$. По аналогии с доказательством теоремы 2 , получаем

$$
\begin{aligned}
\mathbf{P}\left\{\gamma_{n+1}>t+s_{0} \mid \widehat{\mathscr{F}}_{s_{0}}\right\} & \leqslant e^{-\delta t^{\alpha}} \mathbf{E}\left(e^{\delta \gamma_{n+1}^{\alpha}} \mid \widehat{\mathscr{F}}_{s_{0}}\right) \\
& \leqslant e^{-\delta t^{\alpha}} C^{\delta n}\left[\mathbf{E}\left(1+e^{\left|X_{s_{0}}\right|^{\beta}}+e^{\left|Y_{s_{0}}\right|^{\beta}} \mid \widehat{\mathscr{F}}_{s_{0}}\right)\right]^{\delta}
\end{aligned}
$$

При достаточно малом $\delta>0$

$$
\mathbf{P}\left\{L_{s_{0}}>t+s_{0} \mid \widehat{\mathscr{F}}_{s_{0}}\right\} \leqslant C e^{-\delta t^{\alpha}}\left[\mathbf{E}\left(1+e^{\left|X_{s_{0}}\right|^{\beta}}+e^{\left|Y_{s_{0}}\right|^{\beta}} \mid \widehat{\mathscr{F}}_{s_{0}}\right)\right]^{\delta}
$$


Отсюда, по теореме 3 и лемме 4 ,

$$
\begin{aligned}
\beta^{x}(t) & \leqslant \mathbf{E}_{x} \mathbf{P}\left\{L_{s_{0}}>t+s_{0} \mid \widehat{\mathscr{F}}_{s_{0}}\right\} \\
& \leqslant C e^{-\delta t^{\alpha}} \mathbf{E}_{x}\left[\mathbf{E}\left(1+e^{\left|X_{s_{0}}\right|^{\beta}}+e^{\left|Y_{s_{0}}\right|^{\beta}} \mid \widehat{\mathscr{F}}_{s_{0}}\right)^{\delta}\right] \\
& \leqslant C e^{-\delta t^{\alpha}} \mathbf{E}_{x}\left[\mathbf{E}\left(1+e^{\left|X_{s_{0}}\right|^{\beta}}+e^{\left|Y_{s_{0}}\right|^{\beta}} \mid \widehat{\mathscr{F}}_{s_{0}}\right)^{\delta / \delta}\right]^{\delta} \\
& \leqslant C e^{-\delta t^{\alpha}}\left[\mathbf{E}_{x}\left(1+e^{\left|X_{s_{0}}\right|^{\beta}}+e^{\left|Y_{s_{0}}\right|^{\beta}}\right)\right]^{\delta} \\
& \leqslant C e^{-\delta t^{\alpha}}\left(1+\widehat{C}+\widetilde{C}\left(1+e^{|x|^{\beta}}\right)\right)^{\delta} \leqslant C e^{-\delta t^{\alpha}}\left(1+e^{\delta|x|^{\beta}}\right) .
\end{aligned}
$$

Теорема 4 доказана.

Автор выражает глубокую благодарность А. Ю. Веретенникову за постановку задачи и постоянное внимание к работе.

\section{СПИСОК ЛИТЕРАТУРЫ}

1. Veretennikov A. Yu. On polynomial mixing bounds for stochastic differential equation. - Stochastic Process. Appl., 1996, v. 70, p. 115-127.

2. Veretennikov A. Yu. On polynomial mixing and convergence rate for stochastic difference and differential equations. - Preprint № 393. Berlin: WIAS, 1998.

3. Веретенников $A$. Ю. Об ощенках скорости перемешивания для стохастических уравнений. - Теория вероятн. и ее примен., 1987, т. XXXII, в. 2, с. 299-308.

4. Веретенников $A$. Ю. Об оценках скорости перемешивания для марковских прощессов. - Литов. матем. сб., 1991, т. 31, № 1, с. 40-49.

5. Хасьмияский Р. З. Устойчивость систем дифференциальных уравнений при случайных возмушениях их параметров. М.: Наука, 1969, $367 \mathrm{c}$.

6. Крылов Н. В. О стохастических интегральных уравнениях Ито. - Теория вероятн. и ее примен., 1969, т. XIV, в. 2, с. 340-348.

7. Крылов Н.В. О выделении марковского процесса из марковской системы процессов и построении квазидиффузионных процессов. - Изв. АН СССР, матем., 1973 , т. 37, № 3, с. 691-708.

8. Крылов H. В., Сафоков М. В. Об одном свойстве решений параболического уравнения с измеримыми коэффициентами. - Изв. АН СССР, матем., 1980, т. 44, № 1, c. 161-175.

9. Aspandiiarov S., Iasnogorodski R., Menshikov M. Passage-time moments for nonnegative stochastic processes and an application to reflected random walks in a quadrant. - Ann. Probab., 1996, v. 24, № 2, p. 932-960.

10. Ganidis H., Roynette B., Simonot $F$. Convergence rate of some semi-groups to their invariant probability. - Stochastic Process. Appl., 1999, v. 79, p. 243-263.

11. Калаиников В. В. Свойство $\gamma$-возвратности для марковских последовательностей. - Докл. АН СССР, 1973, т. 213, № 6, с. 1243-1246.

12. Menshikov M., Williams $R . J$. Passage-time moments for continuous non-negative stochastic processes and applications. - Adv. Appl. Prob., 1996, v. 28, p. 747-762.

13. Meyn S.P., Tweedie R. L. Markov Chains and Stochastic Stability. Berlin: SpringerVerlag, 1993.

14. Sil'vestrov D.S. Recurrence relation for generalized hitting times for semi-Markov processes. - Ann. Appl. Probab., 1996, v. 6, № 2, p. 617 649.

15. Tuominen P., Tweedie $R$. L. Subgeometric rates of convergence of $f$-ergodic Markov chains. - Adv. Appl. Probab., 1994, v. 26, p. 775-798.

16. Asmussen S. Applied Probability and Queues. New York: Wiley, 1987. 